\title{
Computer-assisted surgery navigation of pedicle screw insertion by standardized trainees
}

LIU Ming-yong, ZHANG Liang, ZHAO Jian-hua, LIU Peng*

Department of Spine Surgery, Daping Hospital, Third Military Medical University, Chongqing, 400042, China

Abstract: Objective To assess the precision and efficiency computer-assisted surgery navigation of pedicle screw insertion by standardized trainees. Methods From September 2013 to June 2016, 360 thoracic pedicle screws were inserted into 100 patients by standardized trainees $(n=30)$. Screws on the left side were inserted by hands (control group), while the other side were inserted under the guidance of computer-assistant navigation system (guided group). The insertion time and volume of blood lost by each screw were retrospectively analyzed. The precision ratio was assessed by computerized tomography (CT) scan after operation. Results The time consumed in screw insertion was significantly shorter in the guided group than that in the control group. The blood lost volume was lesser in the guided group. Post-operation CT scan revealed higher precision in the guided group than that in the control group. Conclusion Computer-assistant navigation system facilitates the learning of standardized trainees, and reduces the time-consuming and bleeding in thoracic pedicle screw insertion.

Orthodontics training is the most important low-grade physician training in the orthopedic department ${ }^{1,2}$. Due to the complexity of spinal anatomy, professional required and high risk of spinal surgery, thoracic pedicle screw implantation has become a very demanding technique. Furthermore, the spine surgery learning is not conducive to the trainees. Since 2013, our department started to use the computer-assisted three-dimensional navigation technology to guide the implantation of screws ${ }^{3}$. We found that the computer-assisted navigation technology has greater advantage when compared with the traditional hand-implanted technology.

Key words: Computer-assisted surgery navigation; pedicle screw insertion ;standardized trainees

\section{Materials and methods}

\subsection{General Information}

Copyright (C) 2017 LIU Ming-yong, et.al.

doi: http://dx.doi.org/10.18686/aem.v6i1.81

This is an open-access article distributed under the terms of the Creative Commons Attribution Unported License

(http://creativecommons.org/licenses/by-nc/4.0/), which permits unrestricted use, distribution, and reproduction in any medium, provided the original work is properly cited.

A total of 30 resident physicians participated the standardized training in our department from September 2013 to June 
2016. This study includes 100 patients, where 62 are males and 38 are females. The age of the patients ranged from 30 to 65 years old. Body mass index (BMI) ranged from 18 to $30 \mathrm{~kg} / \mathrm{m}^{2}$. All patients received the implantation of thoracic pedicle screw and 360 thoracic pedicle screws were implanted. Screws on the left side were inserted by hands (control group) and the right side were inserted under the guidance of computer-assistant navigation system (guided group). Exclusion criteria includes coagulation disorders, systemic infection, severe osteoporosis and other complications. Informed consent was obtained from patients.

\subsection{Surgical methods}

Operations were performed under general anesthesia. Pedicle screw was implanted according to the preoperative setting. Meanwhile, the operation time and bleeding volume were recorded.

The control group: the transverse midline connection and the lateral edge of the facet joint were the entry point. X-ray was used as reference to determine the angle of inclination and cohesion. The pedicle screws were implanted into the correct position.

Guided group: according to the preoperative CT scan, tracking device and navigation system, surgery was conducted under the guidance of the three-dimensional navigation to determine the pedicle axial projection point, head tilt and cohesion angle. The pedicle screws were implanted.

The C-arm X-ray was used to obtain the lateral X-ray, and CT scan was performed to check the pedicle screw position.

\subsection{Observation indicators}

The period of pedicle screw implantation and intraoperative blood loss volume were recorded. The position of the pedicle screw is confirmed by CT scan and is divided into four grades: A, in the pedicle; B, breakthrough the cortex $<2$ $\mathrm{mm}$; breakthrough the cortex 2-4 mm; D, breakthrough the cortex $>4 \mathrm{~mm}^{4}$.

\subsection{Statistical analysis}

SPSS18.0 statistical software analysis was used to analyze the data. Measurement data is presented as mean \pm standard deviation ( $\mathrm{x} \pm \mathrm{SD}$ ). Comparison between groups was analyzed using $t$ test, while the count data between groups were compared using chi-square test. $P<0.05$ for the difference shows statistically significant.

\section{Results}

\subsection{Navigation assistance reduces the time and amount of bleeding}

A total of 30 trainees were enrolled in this study and 360 screws were implanted. Two groups of patients show no significant difference in gender, age and BMI. In the guided group, the screw implantation period and the amount of bleeding were significantly lower than that of the control group. The mean screw implantation period was significantly reduced from $15.3 \mathrm{~min}$ to $10.7 \mathrm{~min}$ in the control group, while the amount of bleeding significantly decreased from $20.6 \mathrm{~mL}$ to $10.4 \mathrm{~mL}$ (Table 1). A significant improvement was observed in the quality of the guided screw implantation in the postoperative CT scan compared with the control group (Table 3). According to the follow-up records of all patients, no screws resulted in any neurological or vascular complications. 
Table 1. Comparison of screw implantation period and bleeding volume.

\begin{tabular}{cccc}
\hline Groups & $\begin{array}{c}\text { Number of } \\
\text { cases }\end{array}$ & $\begin{array}{c}\text { Screw implantation period } \\
(\mathrm{min})\end{array}$ & $\begin{array}{c}\text { Bleeding volume } \\
(\mathrm{mL})\end{array}$ \\
\hline Control group & 180 & $15.3 \pm 1.5$ & $20.6 \pm 15.0$ \\
Guided group & 180 & $10.7 \pm 1.8$ & $10.4 \pm 4.5$ \\
$P$ & & 0.0291 & 0.0408 \\
\hline
\end{tabular}

2.2 Navigation assistance significantly reduces the error rate of screw implantation

The accuracy of pedicle screw implantation was determined through the CT scan. The results showed that quality level of screw implantation in the guided group was significantly higher than that of the control group (Table 2). According to the follow-up records of all patients, no screws resulted in any neurological or vascular complications.

Table 2. Comparison of quality level of screw implantation in two groups.

\begin{tabular}{ccccc}
\hline & \multicolumn{4}{c}{ Quality level of screw implantation } \\
Group & A & B & C & D \\
\hline Control group & 150 & 14 & 10 & 6 \\
Guided group & 167 & 8 & 2 & 0 \\
$\chi^{2}$ & 8.181 & & & \\
$P$ & 0.0424 & & & \\
\hline
\end{tabular}

\section{Discussions}

Thoracic pedicle screw implantation is a key technique of spinal surgery which resulting in high surgical risk due to the presence of spinal cord and large blood vessels in the thoracic spine. Training and teaching demonstration of pedicle screw implantation is difficult. How to master this technique quickly, accurately and is a thorny problem facing in the clinical physician training. We found that computer-assisted navigation technology can significantly shorten the operation period, reduce the amount of blood loss and reduce the error during implantation of screws ${ }^{5-8}$. It is suggested that intraoperative navigation can significantly shorten the learning curve, reduce the time of screw implantation, reduce the amount of bleeding, improve the accuracy of pedicle screw implantation and avoid the risk of culture.

\section{References}

1. Fang Y. Medical graduate students on the standardization of resident training awareness, attitudes and countermeasures (Dissertation). Fudan University, 2016.

2. Ma P, Ma X, Xu X, Shi L, Chen Mai. Exploration of establishing management system of residents' standardization 
training. Progress in Modern Biomedicine, 2015, 15(36): 7187-7189. doi: 10.13241/j.cnki.pmb.2015.36.051

3. Huang Y. Computer-aided navigation technology for clinical research (Dissertation). Suzhou University, 2014.

4. Xu L, Gu R, Zhu Q, et al. Prospective contrast study of vertebrae pedicle screw fixation technology for middle-upper thoracic fracture and dislocation. Chinese Journal of Bone and Joint Injury, 2010, 25(9): 778-780.

5. Bandiera S, Ghermandi R, Gasbarrini A, Barbanti Brodano G, Colangeli S, Boriani S. Navigation-assisted surgery for tumors of the spine. Eur Spine J, 2013; 22 Suppl 6: S919-24.

6. Mason A, Paulsen R, Babuska JM, Rajpal S, Burneikiene S, Nelson EL, Villavicencio AT. The accuracy of pedicle screw placement using intraoperative image guidance systems. Journal of Neurosurgery. Spine, 2014; 20(2): 196-203

7. Moses ZB, Mayer RR, Strickland BA, Kretzer RM, Wolinsky JP, Gokaslan ZL, Baaj AA. Neuronavigation in minimally invasive spine surgery. Neurosurgical Focus, 2013; 35(2): E12

8. Liu YJ, Tian W, Liu B, Li Q, Hu L, Li ZY, Yuan Q, Lu YW, Sun YZ. Comparison of the clinical accuracy of cervical (C2-C7) pedicle screw insertion assisted by fluoroscopy, computed tomography-based navigation, and intraoperative three-dimensional C-arm navigation. Chin Med J, 2010; 123(21): 2995-8. 